\title{
Native nucleic acid electrophoresis as an efficient alternative for genotyping method of influenza virus
}

\author{
Beata Pajak ${ }^{1 凶}$ and Krzysztof Lepek² \\ 'Electron Microscopy Platform, Mossakowski Medical Research Centre, Polish Academy of Sciences, Warsaw, Poland, 2Laboratory of Recombi- \\ nant Vaccines, University of Gdansk and Medical University of Gdansk, Gdańsk, Poland
}

Influenza viruses are the worldwide major causative agents of human and animal acute respiratory infections. Some of the influenza subtypes have caused epidemics and pandemics among humans. The varieties of methods are available for the rapid isolation and identification of influenza viruses in clinical and environmental samples. Since nucleic acids amplification techniques such as RT-PCR have been adapted, fast and sensitive influenza type and subtype determination is possible. However, in some ambiguous cases other, more detailed assay might be desired. The genetic material of influenza virus is highly unstable and constantly mutates. It is known that single nucleotide polymorphisms (SNPs) results in resistance to commercially available anti-viral drugs. The genetic drift of the virus could also result in weakening of immune response to infection. Finally, in a substantial number of patients co-infection with various virus strains or types has been confirmed. Although the detection of co-infection or presence of minor genetic variants within flu-infected patients is not a routine procedure, a rapid and wide spectrum diagnostics of influenza virus infections could reveal an accurate picture of the disease and more importantly, is crucial for choosing the appropriate therapeutics and virus monitoring. Herein we present the evidences that native gel electrophoresis and MSSCP - a method based on multitemperature single strand conformation polymorphism could furnish a useful technique for minor variants, which escape discovery by conventional diagnostic assays.

Key words: gel electrophoresis, SSCP, influenza, minor genetic variants

Received: 27 May, 2014; revised: 18 August, 2014; accepted: 20 August, 2014; available on-line: 01 September, 2014

\section{THE RATIONALE FOR INFLUENZA VIRUS MOLECULAR DIAGNOSTICS}

Recent outbreaks of influenza have shown the necessity to improve diagnostic tests and to develop new methods for fast virus detection, which is crucial for early and efficient implementation of control strategies. The most popular molecular techniques for infection detection and monitoring are based on the amplification of the specific nucleic acid sequences by polymerase chain reaction (PCR or Real Time PCR or multiplex PCR), ligase chain reaction and nucleic acid sequence-based amplification (NASBA), or microarray technology (Lau et al., 2006; Belak et al., 2009; Caliendo et al., 2011).
Important hallmark of influenza virus is a potent ability to undergo antigenic change, which occurs via two ways: antigenic drift and antigenic shift. The first process is gradual and relatively continuous change in the viral haemagglutinin (HA) and neuraminidase (NA) proteins. It results from the accumulation of point mutations in the HA and NA genes sequence during viral replication, leading to a new virus strain formation (Carrat et al., 2007). Influenza virus can also undergo a more profound change called antigenic shift. A shift can occur when new $\mathrm{HA}$ or/and NA proteins are formed via the reassortment of species-specific viruses or when an animal influenza virus infects a human directly, or a non-human virus is passed through a few animal species (e.g. birds, pigs) to humans. Such changes could appear unpredictably and a new virus can become a big threat to human beings. Thus, genetic analysis of influenza virus genes (especially the HA and NA genes) should be used to define the antigenic characteristics of the novel influenza virus strains and subtypes (Noah et al., 2013). Furthermore, the genetic analyses are an useful tool for virus evolution monitoring as well as for the determination of the relatedness between isolates from different geographic regions.

Influenza virus evolution may occur in the patient occasionally, when two viruses of different subtypes meet in the cell of an infected person and mix together their genetic material. A newly created hybrid viruses usually do not survive for long, but those that infect more than one person are known as "the circulating recombinant forms" or CRFs. Such process has been described mainly for HIV-infected patients (Feng et al., 2014). However, in 2009 Canadian physicians reported the case of a new flu virus formation after a child had become co-infected with two influenza strains - pandemic A/ H1N1 and seasonal A/H3N2 (http://www.cbc.ca/ news/canada/toronto/toddler-infected-with-two-influenza-strains-1.1092904). Variability could also be generated upon virus replication and an emerging single nucleotide polymorphisms in HA or NA genes (SNPs) might be associated with the antiviral resistance. Hitherto, neither commercial detection assays for early detection of newlyborne influenza minor genetic variants nor, rapid identi-

e-mail: bepaj@wp.pl

Abbreviations: SNP, single nucleotide polymorphism; MSSCP, Multitemperature single strand conformation polymorphism; NASBA, nucleic acid sequence-based amplification; HA, haemagglutinin; NA, neuraminidase; CRFs, circulating recombinant forms; SSCP, single strand conformation polymorphism; Ab, antibody; ARDS, acute respiratory distress syndrome; RSV, respiratory syncytial virus; $\mathrm{CoV}$, coronavirus; RFLP, restriction fragment length polymorphism; MALDI-TOF MS, matrix-assisted laser desorption ionization-time of flight mass spectrometry. 


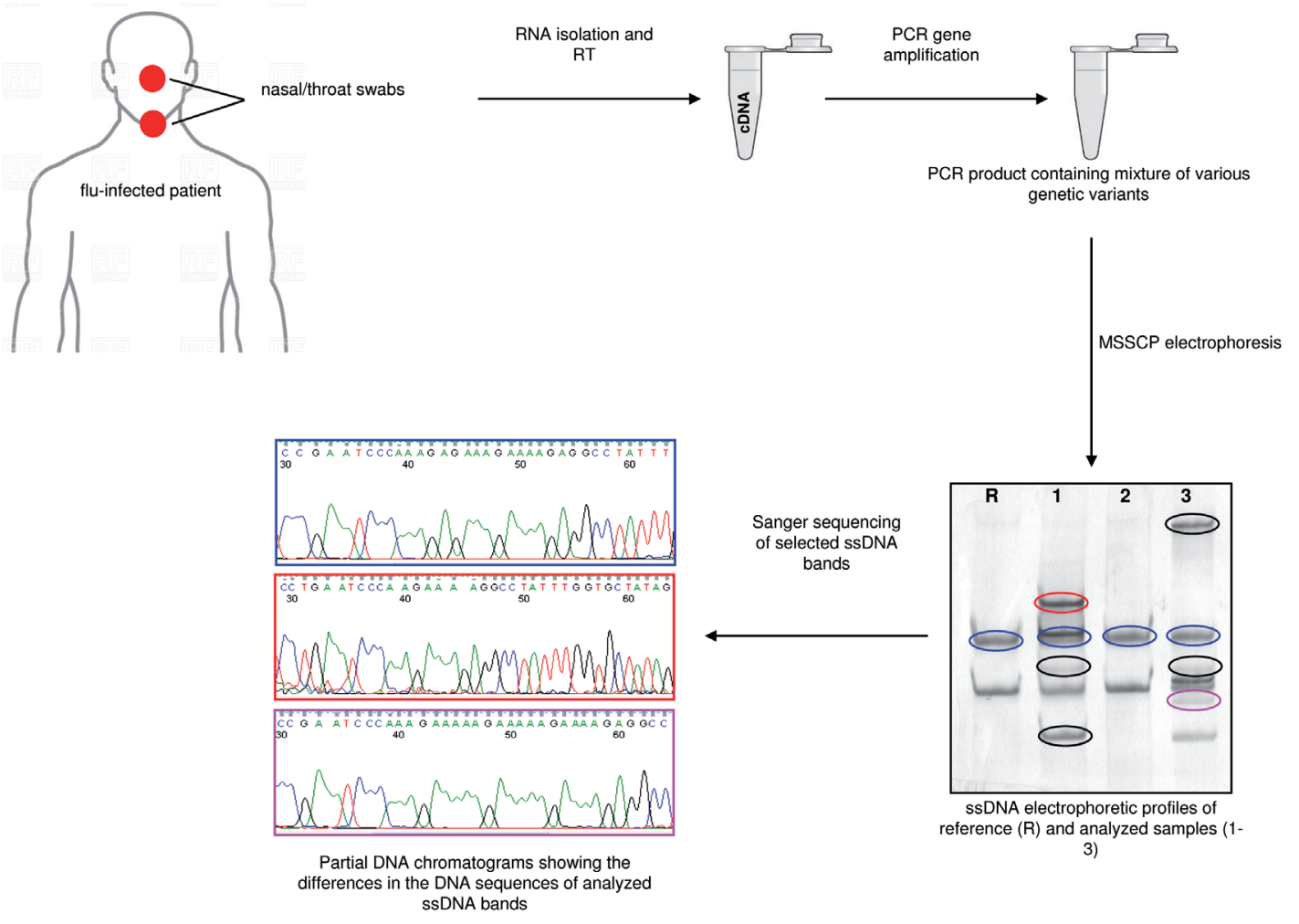

Figure 1. Schematic illustration of influenza virus genotyping via MSSCP analysis.

The nasal or throat swabs from flu-infected patients are collected and viral RNA is isolated. Based on isolated RNA, cDNA is synthesized and is used as a template for PCR amplification of analyzed HA or NA gene fragment. Next, PCR products, as well as reference sample, are separated via native MSSCP electrophoresis at optimal temperature and polyacrylamide (PA) conditions. When MSSCP reveals the presence of additional ssDNA bands suggesting the presence of minor genetic variants within the sample, ssDNA bands are cut off the gel, DNA is recovered and re-amplified. At the same time, the ssDNA bands corresponding to reference electrophoretic profile are also analyzed. Next, PCR products obtained from single ssDNA bands are Sanger sequenced. Obtained DNA chromatograms are analyzed and mutated positions differentiating reference genotype and minor genetic variants are identified.

fication of co-infected patients are available. Herein we propose MSSCP electrophoretic technique as an alternative for the sensitive and rapid discovery and the detection of minor genetic variants, which could be missed by the conventional diagnostics.

\section{NATIVE ELECTROPHORESIS OF NUCLEIC ACIDS}

Native electrophoresis of single stranded nucleic acids - single strand conformation polymorphism (SSCP), is a popular molecular biology analysis, separating PCR products with regard to their sequence. A single nucleotide change in a particular double-stranded DNA sequence cannot be distinguished by electrophoresis, because the physical properties of the double strands are almost identical for both alleles. However, after denaturation, single-stranded DNA undergoes a 3-dimensional folding and may take a unique conformational state based on its DNA sequence. The difference in shape between two DNA strands with different sequences can cause them to migrate differently on an electrophoresis gel, even though the number of nucleotides is the same, showing thus single strand CP (Larsen et al., 2001). The powerful means for mutations or polymorphisms detection via SSCP have restricted electrophoretic conditions, since single-stranded DNA mobility is dependent on temperature and pH (Ren \& Ueland, 1999). MSSCP electrophoresis is performed in gradually reduced, strictly controlled temperature for optimal separation of all conformers present in the sample. MSSCP displays higher sensitivity and reproducibility of distinct ssDNA separations in comparison to classical SSCP. Its advantages were previously demonstrated in oncology and virology research (Krawczyk et al., 2013; Rybicka et al., 2014). We have shown that MSSCP analysis differentiated $\mathrm{A}(\mathrm{H} 1 \mathrm{~N} 1)$ influenza isolates and identified minor genetic variants undetectable by routine procedures (Pajak et al., 2011; Lepek et al., 2014). Schematic representation of influenza diagnostic with MSSCP procedure is illustrated on Fig. 1.

\section{IDENTIFICATION OF INFLUENZA CO-INFECTIONS}

Mixed infections are the main source of reassortants, and although there are confirmed cases of cocirculation of pandemic $\mathrm{A}(\mathrm{H} 1 \mathrm{~N} 1)$, seasonal $\mathrm{A}(\mathrm{H} 1 \mathrm{~N} 1)$ and $\mathrm{A}(\mathrm{H} 3 \mathrm{~N} 2)$ viruses, only a few reports showed mixed infections in patients (Ducatez et al., 2010; Lee et al., 2010; Liu et al., 2010; Ghedin et al., 2011). In 2011 we evaluated the MSSCP approach on 2009 pandemic influenza A-positive clinical specimens collected from flu-diagnosed patients in Poland (Pajak et al., 2011). Using RNA isolated from nasal and throat swabs we amplified 23 samples representing partial HA gene sequence, containing variable region of seasonal and pandemic A(H1N1) strains. Along with clinical samples, reference seasonal (A/Bris- 
bane/59/2007) and pandemic (A/Gdansk/037/2009) isolates were analyzed. Next, PCR products were separated at $15-10-5^{\circ} \mathrm{C}$ electrophoretic temperature cycle and silver stained. MSSCP separation clearly differentiated two reference $\mathrm{A}(\mathrm{H} 1 \mathrm{~N} 1)$ strains, which showed distinct ssDNA pattern on polyacrylamide gel. The majority of clinical samples showed electrophoretic profile characteristic for pandemic A(H1N1) virus, which was in agreement with previous, routine RT-PCR-based diagnostics. However, four samples contained in addition ssDNA bands characteristic of seasonal A(H1N1) strain (Pajak et al., 2011), suggesting co-infections. Noteworthy, previously performed standard diagnostic procedures, qualified those 3 samples as $\mathrm{A}(\mathrm{H} 1 \mathrm{~N} 1)$ pandemic and 1 sample as a seasonal strain-infected. When analyzed via direct Sanger sequencing, obtained sequences were in agreement with RT-PCR results, however, when looking more closely on DNA chromatograms, in co-infected samples the presence of minor double picks in strain-specific HA region was observed. Thanks to MSSCP analysis, ssDNA bands corresponding to pandemic and seasonal $\mathrm{A}(\mathrm{H} 1 \mathrm{~N} 1)$ strains were separated, then cut out from the gel, DNA was eluted and re-amplified. Sanger sequencing of single ssDNA bands confirmed that some of ssDNA bands contained pandemic-, whereas other - seasonal-specific HA gene sequence (Pajak et al., 2011). Interestingly, we did not observe any marked differences in the nucleotide sequences of pandemic and seasonal strains between patients with a dual and those with a single infection.

Described results proved that co-infection is a frequent phenomenon in influenza caused diseases. About $17 \%$ of Polish flu-infected patients were both pandemic and seasonal $\mathrm{A}(\mathrm{H} 1 \mathrm{~N} 1)$ strain-positive. The importance of MSSCP application fully illustrates identification of minor, highly pathogenic pandemic A(H1N1) flu variant in the patient, primary diagnosed as a less pathogenic seasonal A(H1N1) flu-infected. Bearing in mind the risk of highly pathogenic pandemic influenza virus appearance and potential consequences of its global spread, efficient and early detection of minor flu variants is extremely important.

\section{VISUALIZATION OF INFLUENZA ANTIGENIC DRIFT}

Genomic plasticity allows the influenza virus to adapt rapidly through mutations to a wide range of selective pressure. Therefore, characterizing viral genetic variability with MSSCP technology may provide insights into viral population evolution. In our recent studies we have characterized pandemic influenza A(H1N1) circulating in Taiwan during the 2009-2011 outbreak (Lepek et al., 2014). Similarly to our previous work, we analyzed a partial sequence of HA gene, containing region differentiating pandemic and seasonal flu. Consequently, we found that 5 out of 19 Taiwan flu isolates, diagnosed previously with routine RT-PCR as A(H1N1)pdm09, had electrophoretic profiles distinct from pandemic reference ssDNA pattern. New electrophoretic profiles were also not typical for seasonal A(H1N1) strain. More detailed molecular analysis demonstrated the presence of several point mutations, which modulated electrophoretic mobility of analyzed HA gene fragment. Sanger sequencing of DNA fragments obtained from ssDNA bands showed that 5 samples contained different sets of SNPs. Majority of SNPs represented synonymous changes and did not affect amino acid sequence of HA protein. Bioinformatical analysis allowed us to localize positions of detected SNPs in HA protein structure. Seven point mutations were situated in the $\mathrm{E}$ epitope region, but only two, at positions 65 and 66, were in $\mathrm{HA}-\mathrm{Ab}$ binding domain. SNP described in our paper, leading to substitution E66K emerged as a potential immune-response modifying agent, since substitution of glutamic acid with lysine could modulate the chemical bond between $\mathrm{HA}$ and neutralizing $\mathrm{Ab}$ (Lepek et al., 2014). It is also worth mentioning that the observed substitutions present in the E epitope of H1 when they become widespread may affect vaccine effectiveness. Interestingly, Taiwan flu isolates obtained in 2009 remained closely related to pandemic reference strain. New SNPs were present only in 2010 and 2011 isolates, suggesting geographically limited virus evolution.

Importantly, genetic modifications of A(H1N1)pdm09 influenza described above were not detected when analyzed by routine tests. Early detection of minor immuneresistant, potentially highly-pathogenic influenza strains could be important for virus monitoring and optional patients isolation.

\section{MSSCP ANALYSIS IN CLINICAL DIAGNOSTICS OF ARDS PATIENT}

In some cases, respiratory tract complications may result from co-infection with various viral pathogens. Our recent studies showed that MSSCP could be applied also in advanced diagnostic procedures in severe disorder progression with uncertain cause of the infection (Niemcewicz et al., 2013). Also in 2013 Stefanska et al. described a few cases of severe respiratory tract disease caused by mixed infection with influenza virus (IV) type $\mathrm{A}$ and respiratory syncytial virus (RSV) type A or coronavirus (CoV) OC43 or parainfluenza type 3, or a few of them together (Stefanska et al., 2013). Differentiation of particular viruses can be done with multiplex PCR. However, if a few strains of the same virus are present, MSSCP seems to be suitable.

In 2013, in a 60-year old patient acute respiratory distress syndrome (ARDS) was diagnosed with serious infection course. Performed laboratory tests detected the presence of $\mathrm{A}(\mathrm{H} 1 \mathrm{~N} 1) \mathrm{pdm} 09$ virus. However, due to the fatal outcome, RT-PCR product was also analyzed by MSSCP method to exclude possible co-infections with other viruses. As a reference sample, the RT-PCR product obtained with a previously confirmed case of A(H1N1) infection was used. Clinical samples were separated with the use of MSSCP electrophoresis and compared with ssDNA pattern of reference pandemic and seasonal A(H1N1) samples. MSSCP method confirmed that both clinical samples showed ssDNA bands pattern characteristic for $\mathrm{A}(\mathrm{H} 1 \mathrm{~N} 1) \mathrm{pdm} 09$ influenza strain, with a few additional ssDNA bands which could be attributed to co-infection with seasonal A(H1N1) strain or represented another unknown genetic variant (Niemcewicz et al., 2013). The sequences of ssDNA showed that the PCR mixture contained human DNA, corresponding to human chromosome $21 \mathrm{q}$, section $15 / 105$, and that human DNA was efficiently amplified with primers designed for Real-Time A/H1N1 virus detection (Niemcewicz et al., 2013). In conclusion, ARDS syndrome was caused by an immune deficiency of the patient rather then the virus co-infection. That con- 
clusion would not have been drawn without MSSCP analysis. Furthermore, MSSCP-based detection of human material in the viral RT-PCR products points to extremely important problem of false-positive results, resulting in a patient's being included into unnecessary treatment.

\section{MSSCP IN OTHER VIRAL INFECTIONS RESEARCH AND DIAGNOSTICS}

Coexistence of numerous virus variants and quasispecies is a common phenomenon in viral infections. The emergence of drug-resistant mutants is a serious clinical problem in the course of $\mathrm{HBV}$ - and $\mathrm{HCV}$-infected treatments. High replication rate and lack of proofreading activity of the viral polymerase enzyme result in large amount of SNPs within viral sequence. Moreover, sequential therapy with different drugs leads to the accumulation of resistance mutations and, as a consequence, multidrug-resistant viral strain formation (Rybicka et al., 2014). New variants appearing during $\mathrm{HBV}$ or $\mathrm{HCV}$ infection are often more viable, spread rapidly in the liver and become dominant during long-term therapy. It seems obvious that proper treatment strategy should be based on the detection of drug-resistant variants before drug application.

HBV genotyping is usually performed with direct Sanger sequencing of PCR products or restriction fragment length polymorphism (RFLP) or mutationspecific real-time PCR or reverse hybridization or nucleic acids analysis by matrix-assisted laser desorption ionization-time of flight mass spectrometry (MALDITOF MS) (Rybicka et al., 2014). However, most of these methods detect viral mutants that constitute $5 \%$ or even more (20\% in Sanger sequencing) of the viral population. MSSCP assay is much more sensitive and allows to detect even $0.1 \%$ minor variants from viral population (Pajak et al., 2011). To compare the sensitivity of minor HBV genetic variants detection with available methods, Rybicka et al. (2014) performed a series of experiments in clinical samples starting with MALDI-TOF MS and followed by HBV genotyping assays, such as YMDD motif variants detection assay (Inno-Lipa) and MSSCP. According to these results, the most sensitive available method in $\mathrm{HBV}$ diagnostics is MALDI-TOF MS assay that detects minor variants occurring with approximately $1 \%$ frequency. Routine tests revealed results of $40 \%$ and $11 \%$ for YMDD InnoLipa assay and Sanger sequencing, respectively (Rybicka et al., 2014). Important conclusions were drawn by the authors from MSSCP assay in HBV diagnostics. MSSCP showed that $98 \%$ of the HBV is mutated genetic variant, detected by MALDITOF MS technique. It proved that MSSCP technique could provide better results than up-to-date standard procedures. Bearing in mind the limited access to mass spectrometry equipment and significant costs of MALDI-TOF analysis, the application of MSSCP electrophoresis, even if more laborious, appears as an excellent alternative for more sensitive HBV genotyping assays.

Viral quasispecies are also a serious obstacle in successful HCV and HIV infection treatments (Herring et al., 2005; Zheng et al., 2013). We are convinced that MSSCP is a universal solution, whenever minor genetic variants detection is desired.

\section{SUMMARY}

Minor variants have significant implications in quasipecies evolution and viral infection progression. However, the accurate detection of minor genetic viral variants by routine diagnostics methods is often hampered by errors because of their limited sensitivity. Herein we presented results proving that the use of multitemperature native electrophoretic separation (MSSCP) of nucleic acids allowed to detect already known, as well as to discover novel influenza genetic variants in clinical samples. The MSSCP technique provides an inexpensive, convenient, and sensitive method for determining sequence variation and to differentiate influenza strains, and is particularly suitable for the epidemiological studies or a fast screening and typing tool when dealing with a large number of samples. We believe that early detection of potentially highly-pathogenic or drug-resistant viral quasispecies should be a routine procedure in viral diagnostics, which will enable clinical laboratories to provide physicians with new and important information for optimal treatment.

\section{REFERENCES}

Belák S, Kiss I, Viljoen GJ (2009) New developments in the diagnosis of avian influenza. Rev Sci Tech 28: 233-243.

Caliendo AM (2011) Multiplex PCR and emerging technologies for the detection of respiratory pathogens. Clin Inf Dis 52 (Suppl 4): S326S330.

Carrat F, Flahault A (2007) Influenza vaccine: the challenge of antigenic drift. Vaccine 25: 6852-6862.

Ducatez MF, Sonneberg S, Hall RJ, Peacey M, Ralston J, Webby RJ, Huang QS (2010) Genotyping assay for the identification of 20092010 pandemic and seasonal H1N1 influenza virus reassortants. $J$ Virol Methods 168: 78-81.

Feng Y, Wei H, Hsi J, Xing H, He X, Liao L, Ma Y, Ning C, Wang N, Takebe Y, Shao Y(2014) Identification of a Novel HIV Type 1 Circulating Recombinant Form (CRF65_cpx) Composed of CRF01_ $\mathrm{AE}$ and Subtypes B and C in Western Yunnan, China. AIDS Res Hum Retroviruses doi: 10.1089/aid.2013.0233.

Ghedin E, Laplante J, DePasse J, Wentworth DE, Santos RP, Lepow ML, Porter J, Stellrecht K, Lin X, Operario D, nGriesemer S, Fitch A, Halpin RA, Stockwell TB, Spiro DJ, Holmes EC, St George K (2011) Deep sequencing reveals mixed infections with 2009 pandemic influenza A(H1N1) virus strains and the emergence of oseltamivir resistance. I Infec Disease 203: 168-174.

Herring BL, Tsui R, Peddada L, Busch M, Delewart EL (2005) Wide range of quasispecies diversity during promary hepetitis $C$ virus infection. I Virol 79: 4340-4346.

Krawczyk P, Nicos M, Powrozek T, Mlak R, Sawicki M, Jarosz B, Pajak B, Kucharczyk K, Stencel D, Trojanowski T, Milanowski J (2013) Sensitive methods for the detection of an insertion in exon 20 of the HER2 gene in the metastasis of non-small cell lung cancer to the central nervous system. Oncology Lett 6: 1063-1067.

Larsen LA, Christiansen M, Vuust J, Andersen PS (2001) Recent developments in high-throughput mutation screening. Pharmacogenomics 2: 387-399.

Lau LT, Fung YW, Yu AC (2006) Detection of animal viruses using nucleic acid sequence-based amplification (NASBA). Dev Biol (Basel) 126: $7-15$.

Lee N, Chan PK, Lam WY, Szeto CC, Hui DS (2010) Co-infections with pandemic H1N1 and seasonal H3N2 influenza virus. Ann Interm Med 152: 618-619.

Liu W, Li ZD, Tang F, Wei MT, Tong YG, Zhang L, Xin ZT, Ma MJ, Zhang XA, Liu LJ, Zhan L, He C, Yang H, Boucher CA, Richardus JH, Cao WC (2010) Mixed infections of pandemic H1N1 and seasonal H3N2 viruses in 1 outbreak. Clin Infect Dis 50: 1359-1365.

Lepek K, Pajak B, Siedlecki P, Niemcewicz M, Kocik J, Wu HS, Yang JR, Kucharczyk K, Szewczyk B (2014) Genetic diversity of hemagglutinin gene of $\mathrm{A}(\mathrm{H} 1 \mathrm{~N} 1) \mathrm{pdm} 09$ influenza strains isolated in Taiwan and its potential impact on HA-neutralizing epitope interaction. Human Vaccin Immunother 10: 577-585.

Niemewicz M, Pajak B, Michalski A, Kocik J, Kołodziej M, Joniec J, Graniak G, Gawel J, Marciniak-Niemcewicz A, Kucharczyk K, Prystupa A, Witczak A, Lasocki K, Naylor K, Goniewicz M (2013) Acute respiratory distress syndrome (ARDS) in the course of influenza A/H1N1v infection — genetic aspects. Ann Agric Environ Med 20: 711-714. 
Noah DL, Noah JW (2013) Adapting global influenza management strategies to address emerging viruses. Am J Physiol Lung Cell Mol Physiol 305: L108-L117.

Pajak B, Stefanska I, Lepek K, Donevski S, Romanowska M, Szeliga M, Brydak LB, Szewczyk B, Kucharczyk K (2011) Rapid differentiation of mixed influenza A/H1N1 virus infection with seasonal and pandemic variants by Multitemperature single-stranded conformational polymorphism analysis. I Clin Microbiol 49: 2216-2221.

Ren J, Ueland PM (1999) Temperature and $\mathrm{pH}$ effects on single-strand conformation polymorphism analysis by capillary electrophoresis. Hum Mutat 13: 458-463.

Rybicka M, Stalke P, Dreczewski M, Smiatacz T, Bielawski KP (2014) High-throughput matrix-assisted laser desorption ionization-time of flight mass spectrometry as an alternative approach to monitoring drug resistance of hepatitis B virus. J Clin Microbiol 52: 9-14.

Zheng DP, Rodrigues M, Bile E, Nguyen DB, Diallo K, DeVos JR, Nkengasong JN, Yang C (2013) Molecular characterization of ambiguous mutations in HIV-1 polymerase gene: implications for monitoring HIV infection status and drug resistance. PLoS One 8: e77649.

www.cbc.ca/news/canada/toronto/toddler-infected-with-two-influenzastrains-1.1092904 